\title{
RIEMANN-ROCH FOR EQUIVARIANT CHOW GROUPS
}

\author{
DAN EDIDIN AND WILLIAM GRAHAM
}

\section{INTRODUCTION}

The purpose of this paper is to prove an equivariant Riemann-Roch theorem for schemes or algebraic spaces with an action of a linear algebraic group $G$. For a $G$-space $X$, this theorem gives an isomorphism

$$
\left.\tau^{G}: G^{G}(X) \rightarrow \widehat{G^{G}(X)}\right)_{\mathbb{Q}} \stackrel{\simeq}{\rightarrow} \prod_{i=0}^{\infty} C H_{G}^{i}(X)_{\mathbb{Q}} .
$$

Here $\widehat{G^{G}(X)}$ is the completion alon the augmentation ideal of the representation ring $R(G)$, and he groups $C H_{G}^{i}(X)$ are the equivariant Chow groups defined in EG2]. The map $\tau^{G}$ has the same functorial properties as the non-equivariant Riemann-Roch map of [BFM], [Fu, Theorem 18.3] and if $G$ acts freely then $\tau^{G}$ can be identified with the non-equivariant Todd class map $\tau_{X / G}: G(X / G) \rightarrow C H^{*}(X / G)_{\mathbb{Q}}$.

The key to proving this isomorphism is a geometric description of completions of the equivariant Grothendieck group (Theorem 2.1). Besides Riemann-Roch, this result has some purely $K$-theoretic applications. In particular, we prove (Corollary 6.2) a conjecture of Köck (in the case of regular schemes) and extend to arbitrary characteristic a result of Segal on representation rings (Corollary 6.1).

For actions with finite stabilizers the equivariant Riemann-Roch theorem is more precise; it gives an isomorphism between a localization of $G^{G}(X)$ and $\oplus C H_{G}^{i}(X)$ (Corollary 5.1). This formulation enables us to give a simple proof of a conjecture of Vistoli (Corollary 5.2). If $G$ is diagonalizable, then we can express $G^{G}(X)$ in terms of the equivariant Chow groups (an unpublished result of Vistoli, cf. also [To]). Actions with finite stabilizers are particularly important because quotients by these actions arise naturally in geometric invariant theory. In a subsequent paper, we will use these results to express the Todd class map for a quotient of such an action in terms of equivariant Todd class maps, generalizing Riemann-Roch formulas of Atiyah and Kawasaki.

The first author was partially by NSA grant MDA904-97-1-0030 and the M.U. research board. The second author was partially supported by the NSF. 
The main tool of this paper is the approximation of total space of the classifying bundle $E G$ by an open subset $U$ of a representation $V$, where $G$ acts freely on $U$, and $V-U$ is a finite union of linear subspaces. Approximations to $E G$ by open sets in representations were introduced by Totaro in Chow theory [1] , and used in [EG2 to define equivariant Chow groups. However, in these papers, $V-U$ is only required to have large codimension: because Chow groups are naturally graded we can identify $\oplus_{0}^{N} C H_{G}^{i}(X)$ with $\oplus_{0}^{N} C H_{G}^{i}(X \times U)$ as long as codim $(V-U)>N$. Since Grothendieck groups are not naturally graded we need the stronger condition that $V-U$ is a union of linear subspaces. to compare $G^{G}(X)$ with $G^{G}(X \times U)$.

Such $V$ and $U$ can be found for certain types of groups: for finite groups, tori, or Borel subgroups of $G L_{n}$. For such groups, we prove that the $I$-adic completion of $G^{G}(X)$ has geometric description. This fact directly implies the equivariant Riemann-Roch isomorphism for these groups. For general $G$, however, it seems unlikely that such $V$ and $U$ exist, so we must employ a less direct approach. The Riemann-Roch theorem for general $G$ is deduced by embedding $G$ into $G L_{n}$ and then reducing the case of $G L_{n}$ to that of a Borel subgroup. This strategy of is due to Atiyah and Segal for compact groups. If $G$ is connected and reductive then we can obtain the theorem for $G$ directly from the result for a Borel subgroup of $G$, without embedding $G$ in $G L_{n}$.

The necessity of using completions of equivariant Grothendieck groups also goes back to Atiyah and Segal. In our setting it is motivated as follows. For smooth varieties the Todd class map is defined by a power series which, in the non-equivariant case, terminates on any particular variety. However, the equivariant Chow groups of a fixed variety can be nonzero in arbitrarily high degree, so the natural image of the equivariant Todd class map is actually the infinite product $\prod_{i=0}^{\infty} C H_{G}^{i}(X)$. To obtain a Riemann-Roch isomorphism, it is natural to expect that the equivariant Grothendieck groups must also be completed, as is indeed the case. There is one essential difference between these completions. The completion for Chow groups is injective, as it simply replaces a direct sum by a direct product, but on the Grothendieck group side, information is definitely lost by completing (cf. Section 5).

1.1. Contents. The contents of the paper are as follows. In Section 2 we define the completions we need for the main results, and prove a geometric description of these completions for tori and for the subgroup of upper triangular matrices of $G L_{n}$. In Section 3 we construct the equivariant Riemann-Roch map, and prove that it has the same functorial properties as the non-equivariant Riemann-Roch map. Moreover, it 
behaves naturally with respect to restriction to a subgroup (Section 3.2). In Section 3.1 we illustrate the use of this theorem by deriving the Weyl character formula for $S L_{2}$ following [B]. In Section 4 we prove that the Riemann-Roch map induces an isomorphism on the completions. Section 5 contains results, mentioned above, on actions with finite stabilizers, and proves Vistoli's conjecture. Finally, in Section 6 we prove that two naturally defined completions of equivariant $K$-theory are equal, and apply it to prove Köck's conjecture and the result about representation rings.

1.2. Conventions and notation. All groups in this paper are assumed to be linear algebraic groups over an arbitrary field $k$ (i.e. closed subgroup-schemes of $\left.G L_{n}(k)\right)$. Note that in characteristic $p$ such groups need not be smooth over $k$. Representations of $G$ are assumed to be rational, i.e., linear actions of $G$ on finite dimensional $k$-vector spaces.

Free actions By a free action of $G$ on $X$ we mean an action that is scheme-theoretically free, i.e., the action map $G \times X \rightarrow X \times X$ is a closed embedding. An action that is proper and set-theoretically free is free.

Algebraic spaces This paper is written in the language of algebraic spaces. Unless stated otherwise, a space is an equidimensional quasiseparated algebraic space over $k$. One reason to work in this category is that we need quotients of the form $X \times{ }^{G} U$ where $U$ is an open set in a representation of $G$ on which $G$ acts freely. In the category of algebraic spaces, such quotients exist, by a result of Artin [EG2, Proposition 22]). It is possible to work entirely in the smaller category of schemes of finite type over $k$, but some mild technical hypotheses are required to ensure that quotients exist as schemes (EG2, Proposition 23]). Because free actions are proper, if $X$ is separated, so is $X \times^{G} U$.

If $Z$ is a separated algebraic space, then there is a Riemann-Roch map $\tau_{Z}: G(Z) \rightarrow C H^{*}(Z)_{\mathbb{Q}}$ with the same properties as the RiemannRoch map for schemes. This fact (cf. Gi]) can be deduced from the Riemann-Roch theorem for quasi-projective schemes and the existence of Chow envelopes for separated algebraic spaces.

Equivariant Chow groups We will use the notation $A_{k}^{G}(X)$ to refer to the equivariant Chow groups of "dimension" $k$ as defined in [EG2]. However, it is usually more convenient to index by codimension. We write $C H_{G}^{i}(X)$ for the "codimension" $i$ equivariant Chow group: if $X$ has pure dimension $n$, then $C H_{G}^{i}(X):=A_{n-i}^{G}(X)$. The notation $A_{G}^{i}(X)$ to refers to the degree $i$ operational Chow group; when $X$ is 
smooth, $C H_{G}^{i}(X) \simeq A_{G}^{i}(X)$ EG2]. The operational Chow groups have a natural product, and $\oplus_{i=0}^{\infty} C H_{G}^{i}(X)$ is a positively graded module for the graded ring $\oplus A_{G}^{i}(X)$.

The $G$-equivariant Chow ring of a point will be denoted $A_{G}^{*}$. Pullback from a point makes $\oplus_{i}^{\infty} C H_{G}^{i}(X)$ a graded $A_{G}^{*}$-module.

Equivariant $K$-theory We will use the notation $K^{G}(X)$ to refer to the Grothendieck group of $G$-equivariant vector bundles. The Grothendieck group of $G$-equivariant coherent sheaves will be denoted $G^{G}(X)$. Tensor product gives a ring structure on $K^{G}(X)$, and $G^{G}(X)$ is a module for this ring. If $X$ is regular and has the property that every coherent sheaf is the quotient of a locally free sheaf (this holds for regular schemes, but is not known in general for regular algebraic spaces) then a result of Thomason ([Tho3, Corollary 5.8]) says that these groups are isomorphic.

The representation ring $R(G)$ can be identified with $K^{G}(p t)$, and consequently $G^{G}(X)$ has an $R(G)$-module structure. If $A$ is a ring write $G^{G}(X)_{A}=G^{G}(X) \otimes_{\mathbb{Z}} A$, the Grothendieck group with coefficients in $A$, and similarly for Chow groups.

\section{Completions}

In this section we work with a fixed coefficient $\operatorname{ring} A$ and write simply $G^{G}(X)$ for $G^{G}(X)_{A}$, and similarly for Chow groups.

We will use the following completions in the sequel. The graded ring $\oplus A_{G}^{i}(X)$ completes to $\prod_{i=0}^{\infty} A_{G}^{i}(X)$ and $\prod_{i=0}^{\infty} C H_{G}^{i}(X)$ is naturally a module for this ring. We will let $J$ denote the augmentation ideal in $A_{G}^{*}$, and let $\widehat{C H_{G}^{*}(X)}$ denote the completion of the $R$-module $C H_{G}^{*}(X)$ along $J$.

Completing equivariant $K$-theory requires more care since $K^{G}$ is not naturally graded. We complete as follows.

Choose an embedding $G \hookrightarrow G L_{n}$. This induces a homomorphism $R=R\left(G L_{n}\right) \rightarrow R(G)$. Thus $G^{G}(X)$ is an $R$-module. Let $I$ be the augmentation ideal of $R$ (the ideal of virtual representations of $G L_{n}$ of dimension 0 ), and $I_{G}$ the augmentation ideal of $R(G)$. We denote the $I$-adic completion of $G^{G}(X)$ by $\widehat{G^{G}(X)}$.

This definition is convenient for proving results about $G$ by reducing to the case of $G L_{n}$, but it is not obvious that it is independent of the choice of embedding of $G$ into $G L_{n}$. However, this follows from Corollary 6.1, which states that $I$ and $I_{G}$ give the same topology on $R(G)$ whenever $G \hookrightarrow G L_{n}$; hence $I$-adic and $I_{G}$-adic completions are isomorphic. A special case of this is proved in Proposition 2.2; the general case, using the geometric methods of Theorem 2.1, is Corollary 
6.1. In characteristic 0, this result is the same as [Segal, Corollary 3.9]. However, his methods do not extend to characteristic $p$.

2.1. Geometric completions. There is a way of completing equivariant $K$-groups and Chow groups that is more directly related to the definition of equivariant Chow groups. For any representation $V$ of $G$, let $V^{0}$ denote the open subset of points of $V$ on which $G$ acts freely and let $U$ be an open subset of $V^{0}$. A quotient $(X \times U) / G$ exists; such a quotient is usually written as $X \times^{G} U$. Choose $V$ and $U$ such that the codimension of $V-U$ is greater than $k$ (this is always possible); then by definition, $C H_{G}^{k}(X)=C H^{k}\left(X \times^{G} U\right)$.

With this as motivation, let $\mathcal{V}$ be a collection of pairs $(V, U)$ of $G$ modules and invariant open sets with the following properties:

(i) $G$ acts freely on $U$.

(ii) If $\left(V_{1}, U_{1}\right)$ and $\left(V_{2}, U_{2}\right)$ are in $\mathcal{V}$ then there is a pair $\left(V_{1} \oplus V_{2}, U\right)$ in $\mathcal{V}$ such that $U$ contains $U_{1} \times V_{2}$ and $V_{1} \times U_{2}$.

The elements of such a system can be partially ordered by the rule $\left(W, U^{\prime}\right)<(V, U)$ if the $G$-module $V$ can be written as a direct sum $V=W \oplus W^{\prime}$ with $U \supset U^{\prime} \times W^{\prime}$. Suppose that $(V, U)$ is in $\mathcal{V}$; by the homotopy property, we can identify $G^{G}(X)=G^{G}(X \times V)$ and $C H_{G}^{i}(X \times V)=C H_{G}^{i}(X)$. In this way we obtain surjective maps

$$
k_{V}: G_{G}^{0}(X) \rightarrow G^{G}(X \times U)
$$

and

$$
r_{V}: \oplus C H_{G}^{i}(X) \rightarrow \oplus C H_{G}^{i}(X \times U) .
$$

Lemma 2.1. If $\left(W, U^{\prime}\right)<(V, U)$ then $\operatorname{ker} k_{W} \supseteq \operatorname{ker} k_{V}$ and $\operatorname{ker} r_{W} \supseteq$ $\operatorname{ker} r_{V}$.

Proof. Write $V=W \oplus W^{\prime}$. Then $U \supset U^{\prime} \times W^{\prime}$, so we have surjective maps

$$
G^{G}(X) \rightarrow G^{G}(X \times U) \rightarrow G^{G}\left(X \times U^{\prime} \times W^{\prime}\right) \cong G^{G}\left(X \times U^{\prime}\right) .
$$

The first map is $k_{V}$ and the composition is $k_{W}$, proving the first inclusion. A similar argument works for $r_{V}$ and $r_{W}$.

For $\left(W, U^{\prime}\right)<(V, U)$, the lemma implies there is a natural surjective map

$$
G^{G}(X) / \operatorname{ker} k_{V} \rightarrow G^{G}(X) / \operatorname{ker} k_{W}
$$

making these groups into an inverse system indexed by $\mathcal{V}$. A similar statement holds for the Chow groups. Taking the inverse limit we obtain a completion of $G^{G}(X)$. For an arbitrary system $\mathcal{V}$ it is difficult 
to describe this completion. However, there is one situation where we can understand it.

Call a system $\mathcal{V}$ with properties (i) and (ii) above good if it has a third property:

(iii) If $(V, U)$ is in $\mathcal{V}$ then $V-U$ is contained in a finite union of invariant linear subspaces.

Theorem 2.1. Let $\mathcal{V}$ be a good system of representations. Then:

(a) The topology on $G^{G}(X)$ induced by the subgroups ker $k_{V}$ coincides with the $I_{G^{-}}$adic topology. Hence $\lim _{\leftarrow(V, U) \in \mathcal{V}}\left(G^{G}(X) / \operatorname{ker} k_{V}\right)$ is isomor-

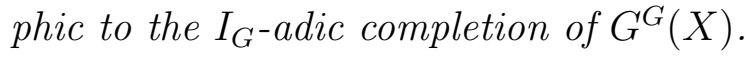

(b) The topology on $C H_{G}^{*}(X)$ induced by the subgroups $\operatorname{ker} r_{V}$ coincides with the J-adic topology. Hence $\lim _{\leftarrow V, U) \in \mathcal{V}} C H_{G}^{*}(X) / \operatorname{ker} r_{V}$ is isomorphic to the J-adic completion of $C H_{G}^{*}(X)$.

The following result shows that for certain classes of groups, good systems exist.

Theorem 2.2. If $G$ is a finite group, a torus or more generally any subgroup of the group of upper triangular matrices in $G L_{n}$, then there exists a system $\mathcal{V}$ of good pairs for $G$.

In particular, if the ground field is algebraically closed then any solvable group has a good system.

The proofs of these theorems will be given in the next 2 subsections. We close with two propositions which will be used in the sequel.

Proposition 2.1. Let $G$ be a subgroup of the group of upper triangular matrices. Then

$$
\widehat{C H_{G}^{*}(X)} \simeq \prod_{i=0}^{\infty} C H_{G}^{i}(X) .
$$

Proof of Proposition 2.1. If $G$ is a subgroup of the upper triangular matrices then $G=T U$ with $T$ a torus and $U$ unipotent. The homotopy property of equivariant Chow groups implies that $C H_{G}^{*}(X)=C H_{T}^{*}(X)$ so we assume $G=T$ is a torus.

Let $M=C H_{G}^{*}(X)$ and $M_{n}=\oplus_{i=n+1}^{\infty} C H_{G}^{i}(X)$. We will show that the filtrations of $M$ given by $\left\{J^{n} M\right\}$ and $\left\{M_{n}\right\}$ give the same topology.

Step 1. For any $n$ we must show there exists $l$ such that $J^{l} \subseteq M_{n}$. Since $J^{l}$ maps $C H_{G}^{i}(X)$ to $C H_{G}^{i+l}(X)$, if $l>n$ the desired inclusion holds.

Step 2. For any $n$ we must show there exists $l$ such that $M_{l} \subseteq J^{n}$. Brion $\left[\mathrm{Br}\right.$, Theorem 2.1] has shown that $C H_{T}^{*}(X)$ is generated as an $A_{T^{-}}^{*}$ module by fundamental classes of $T$-invariant subvarieties. Any such 
fundamental class lies in $C H_{G}^{i}(X)$ for some $i \leq N$, where $N=\operatorname{dim} X$. Hence if $r>\operatorname{dim} X$, then $C H^{r}(G) \subseteq J^{r-N} \cdot\left(\oplus_{i \leq N} C H_{G}^{i}(X)\right)$. Hence if $l>n+N$, we have $M_{l} \subseteq J^{n}$.

Proposition 2.2. If $G=G L_{n}$ and $B$ (resp. T) is the group of upper triangular (resp. diagonal) matrices then

(a) The map $R(B) \rightarrow R(G)=R(B)^{W}$ is finite.

(b) $I_{G L_{n}}$ generates same topology on $R(B)$ (resp. $R(T)$ ) as $I_{B}$ (resp. $\left.I_{T}\right)$.

Proof. The Weyl group of $G L_{n}$ is $S_{n}$ over any field, so by Serre, Example 3.8] $R(G)=R(B)^{S_{n}}$. Thus it suffices to prove the fact for $G=G L_{n}(\mathbb{C})$. The follows from Sega], Proposition 3.2 and Corollary 3.9] applied to maximal compact subgroups $U_{n}$ and $\left(S^{1}\right)^{n}$.

2.2. Proof of Theorem 2.2. First suppose that $G$ is finite. We construct a system as follows: For each representation $V$ let $V^{0}$ be the set of points with trivial stabilizer. Since $G$ is finite, the action is automatically proper. If $V$ and $W$ are representations then $(V \oplus W)^{0} \supset V^{0} \times W \cup V \times W^{0}$. Thus, the collection $\mathcal{V}=\left\{V, V^{0}\right\}$ is a system satisfying (i) and (ii). To see that it is a good system, note that

$$
V-V^{0}=\bigcup_{g \in G, g \neq 1} V^{g}
$$

where $V^{g}$ is the linear subspace fixed by $g$.

Lemma 2.2. Let $H \subset G$ be subgroup. If $\mathcal{V}$ is a good system for $G$, then is also a good system for the restriction of the action to $H$.

Proof. Since properties (ii) and (iii) of a good system are independent of the group action, it suffices to show property (i); i.e. if $(V, U) \in \mathcal{V}$ then $H$ acts freely on $U$. This is clear since the action map $H \times U \rightarrow U \times U$ is the composition of the closed embedding $H \times U \hookrightarrow G \times U$ and the action map $G \times U \rightarrow U \times U$, which we have assumed to be proper.

By the Lemma it suffices to construct a good system for the group $B$ of upper triangular matrices in $G l_{n}$. We will do this as follows: Let $V_{1}$ be the vector space of upper triangular matrices; $B$ acts on $V_{1}$ by left matrix multiplication. Let $U_{1} \subset V_{1}$ be the subset of invertible elements. Then $V_{1}-U_{1}$ is a union of $n$ hyperplanes. Set $V_{k}=V_{1}^{\oplus k}$ and let $U_{k} \subset V_{k}$ consist of the $k$-tuples of $V_{1}$ such that at least one element of the $k$-tuple lies in $U_{1}$. Then $V_{k}-U_{k}$ is a union of linear subspaces. By construction the collection of pairs $\left\{\left(V_{k}, U_{k}\right)\right\}$ satisfies properties (ii) and (iii) of a good system. The action of $B$ on $U_{k}$ has trivial stabilizers, but we must actually check that that the action map $B \times U_{k} \rightarrow U_{k} \rightarrow U_{k}$ is a closed 
embedding. A tuple of matrices $\left(A_{1}, \ldots, A_{k}, B_{1}, \ldots, B_{k}\right) \in U_{k} \times U_{k}$ is in the image of $B \times U_{k}$ if and only if there is a matrix $A \in B$ such that $B_{i}=A A_{i}$ for all $i$. Thus, the image of $B \times U_{k}$ in $U_{k} \rightarrow U_{k}$ is the closed subvariety defined by the equations

$$
\operatorname{det}\left(B_{i}\right) \operatorname{Adj}\left(A_{j}\right)^{t} A_{j}=\operatorname{det}\left(A_{i}\right) \operatorname{Adj}\left(B_{i}\right)^{t} B_{j} .
$$

\subsection{Proof of Theorem 2.1.}

Remark 2.1. Let $(V, U)$ be a pair in our system $\mathcal{V}$ of good representations. As can be seen from the proof below, we only use the fact that $G$ acts with trivial stabilizers (as opposed to freely) an the open set $U \subset V$. However, the freeness of the action will be essential when apply Theorem 2.1 to prove of the Riemann-Roch isomorphism. The reason is that we will need to know that if $X$ is a separated algebraic space then $X \times{ }^{G} U$ is still separated.

Before proving the theorem, we prove several preliminary results.

Lemma 2.3. Let $X$ be a $G$-space which is a union of irreducible components $X_{1}, \ldots, X_{k}$. Then proper push-forward gives a surjection

$$
\oplus G^{G}\left(X_{i}\right) \rightarrow G^{G}(X)
$$

Proof. By induction it suffices to prove the lemma when $X=X_{1} \cup X_{2}$. Let $\tilde{X}$ be the disjoint union of $X_{1}$ and $X_{2}$. Then $G^{G}(\tilde{X})=G^{G}\left(X_{1}\right) \oplus$ $G^{G}\left(X_{2}\right)$. The finite surjective map $\tilde{X} \rightarrow X$ gives a map of localization exact sequences

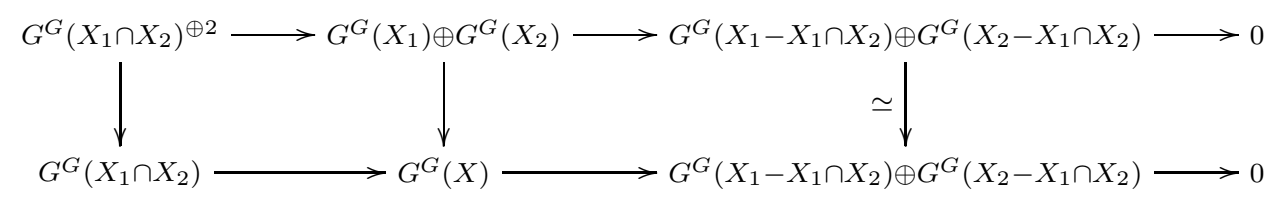

A diagram-chase shows that the map $G^{G}\left(X_{1}\right) \oplus G^{G}\left(X_{2}\right) \rightarrow G^{G}(X)$ is surjective.

Remark 2.2. The analogous statement for non-equivariant theory also holds for higher $K$-theory. However, for higher $K$-theory the only proof we know uses the Brown-Gersten spectral sequence. Since we do not know how to adapt that sequence to equivariant $K$-theory we can not state a result for higher equivariant $K$-theory.

Lemma 2.4. Let $V$ be a representation of $G$ and $L \subsetneq V$ a sub-representation. Let $i: X \times W \rightarrow X \times V$ be the inclusion. Then

$$
i_{*} G^{G}(X \times W) \subset I_{G} G^{G}(X \times V)
$$

where $I_{G} \subset R(G)$ is the augmentation ideal. 
Proof. By the homotopy property of equivariant $G$-theory the pullback $i^{*}: G^{G}(X \times V) \rightarrow G^{G}(X \times L)$ is an isomorphism of $R(G)$-modules. Hence it suffices to show that $i_{*} i^{*} G^{G}(X \times L) \subset I_{G} G^{G}(X \times V)$. By the self-intersection formula,

$$
i^{*} i_{*}(\alpha)=\lambda_{-1}([V / L]) \alpha .
$$

Since $\lambda_{-1}([V / L]) \in I_{G}$, the lemma follows.

Lemma 2.5. Let $Z$ be a separated algebraic space, and let

$\mathfrak{a}_{Z}=\operatorname{ker}(K(Z) \rightarrow \mathbb{Z})$ be the augmentation ideal. Then if $k>\operatorname{dim} Z$, $\mathfrak{a}_{Z}^{k} G(Z)=0$. If $Z$ is not separated then $\mathfrak{a}_{Z}^{k} G(Z)=0$ for $k$ sufficiently large.

Proof. Let $Z$ be a separated algebraic space. Following [Fu, Definition 18.3] we define a Chow envelope $Z^{\prime} \stackrel{p}{\rightarrow} Z$ to be a proper morphism from a quasi-projective scheme $Z^{\prime}$, such that for every integral subspace $V \subset Z$, there is a subvariety $V^{\prime}$ of $X$ such that $p$ maps $V^{\prime}$ birationally to $V$. Using Chow's lemma for algebraic spaces ([Kn, Theorem IV.3.1]), the argument of $[\mathrm{Fu}$, Lemma 18.3] shows that every algebraic space has a Chow envelope (of the same dimension as $Z$ ), and that the proper push-forward $p_{*}: G\left(Z^{\prime}\right) \rightarrow G(Z)$ is surjective.

Since $Z^{\prime}$ is quasi-projective, $F_{Z^{\prime}}^{k} G\left(Z^{\prime}\right)=0([\mathrm{FD}$, Cor. 3.10]) where $F_{Z^{\prime}}^{k}$ is the $k$-th level of the $\gamma$-filtration. Since $p^{*}: K(Z) \rightarrow K\left(Z^{\prime}\right)$ preserves the rank of a locally free sheaf, $p^{*} \mathfrak{a}_{Z}^{k} \subset \mathfrak{a}_{Z^{\prime}}^{k}$. By definition, $\mathfrak{a}_{Z^{\prime}}^{k} \subset F^{k}$, so $p^{*} \mathfrak{a}_{Z}^{k} G\left(Z^{\prime}\right)=0$. Hence, by the projection formula, $\mathfrak{a}_{Z}^{k} p_{*} G\left(Z^{\prime}\right)=0$. Since $p_{*}$ is surjective, the lemma follows.

Even if $Z$ is not separated it has open set $W$ which is a separated scheme. Then $\mathfrak{a}^{k} G^{G}(W)=0$ for $k>\operatorname{dim} Z$. Using the Noetherian induction and the localization sequence we see that $\mathfrak{a}_{W}^{k} G^{G}(Z)=0$ for $k>>0$.

Lemma 2.6. Suppose that $G$ acts freely on an algebraic space $Z$. Then $I_{G}^{k} G^{G}(Z)=0$ for $k>>0$.

Proof. Let $Y=Z / G$ be the quotient algebraic space. Since $Z \rightarrow Y$ is a principal bundle, we have $G^{G}(Z) \simeq G(Y)$. Under this isomorphism, $I_{G}^{l} G^{G}(Z) \subset \mathfrak{a}^{l} G(U)$. The lemma now follows from Lemma 2.6

Proof of Theorem 2.1. We prove only part (a). The corresponding result about Chow groups has essentially the same proof.

To show that the filtrations of $G^{G}(X)$ by the submodules ker $k_{V}$ and by powers of the ideal $I_{G}$ generate the same topology, there are two steps. 
Step 1. We must show that given any pair $(V, U)$, there is an integer $k$ such that $I_{G}^{k} G^{G}(X) \subset$ ker $k_{V}$, or in other words, that $I_{G}^{k} G^{G}(X \times U)=0$. Since $G$ acts freely on $X \times U$ this follows from Lemma 2.6.

Step 2. We must show that a given positive integer $k$, there is a pair $(V, U)$ such that ker $k_{V} \subset I_{G}^{k} G^{G}(X)$. Let $(V, U)$ be any good pair and set $C=V-U$. Then $\operatorname{ker} k_{V}=\operatorname{im}\left(G^{G}(X \times C) \rightarrow G^{G}(X)\right)$. By definition, $C=\bigcup C_{i}$ where $C_{i}$ is contained in a linear subspace $L_{i}$ of $V$, and by Lemma 2.4, $\operatorname{im}\left(G^{G}\left(X \times C_{i}\right)\right) \subset I_{G} G^{G}(X)$. By Lemma 2.3, $\operatorname{im}\left(G^{G}(X \times C)\right)$ is generated by the images of $G^{G}\left(X \times C_{i}\right)$, so $\operatorname{im}\left(G^{G}(X \times C)\right) \subset I_{G} G^{G}(X)$.

Now let $U_{j} \subset V^{\oplus k}$ be the subset where the $j$-th component lies in $U$, and let $D_{j}=V^{\oplus k}-U_{j}$. By induction,

$$
\operatorname{im}\left(G^{G}\left(X \times D_{j}\right)\right) \subset I_{G}^{k} G^{G}\left(X \times V^{\oplus k}\right) .
$$

There is a good pair $\left(V^{\oplus k}, U^{\prime}\right)$ where $U^{\prime}$ contains each $U_{j}$; then

$$
\operatorname{im}\left(G^{G}\left(X \times\left(V^{\oplus k}-U^{\prime}\right)\right)\right) \subset I_{G}^{k} G^{G}(X)
$$

as desired.

\section{Construction of an equivariant Riemann-Roch maP}

All spaces considered here are assumed to be separated.

In this section we construct an equivariant Riemann-Roch map

$$
\tau^{G}: G^{G}(X) \rightarrow \prod_{i=0}^{\infty} C H_{G}^{i}(X)_{\mathbb{Q}}
$$

with the same functoriality as in the non-equivariant case $[\mathrm{Fu}$, Chapter 17]. In addition this map will factor through the completion map $G^{G}(X) \rightarrow \widehat{G^{G}(X)}$.

The results of the previous section are not needed to construct the map but they are used in the next section to show that the map induces an isomorphism $\left.\widehat{G^{G}(X)}\right)_{\mathbb{Q}} \rightarrow \prod_{i=0}^{\infty} C H_{G}^{i}(X)_{\mathbb{Q}}$. To simplify notation we will write $C H_{G}^{i}(X)$ for $C H_{G}^{i}(X)_{\mathbb{Q}}$.

Equivariant Todd classes and Chern characters As in the nonequivariant case, an equivariant vector bundle $E \rightarrow X$ of rank $r$ has Chern roots $x_{1}, \ldots x_{r}$ (obtained via the splitting principle) such that $c_{i}^{G}(E)=e_{i}\left(x_{1}, \ldots x_{r}\right)$. 
Definition 3.1. Let $E$ be a vector bundle with Chern roots $x_{1}, \ldots x_{r}$. Define the equivariant Chern character

$$
c h_{G}: K^{G}(X) \rightarrow \prod_{i=0}^{\infty} A_{G}^{i}(X)
$$

by the formula

$$
c h_{G}(E)=\sum_{i=1}^{r} e^{x_{i}} .
$$

Likewise, define the equivariant Todd class by the formula

$$
\operatorname{Td}^{G}(E)=\prod_{i=1}^{r} \frac{x_{i}}{1-e^{-x_{i}}} .
$$

Because the leading coefficient of $\mathrm{Td}^{G}(E)$ is $1, \mathrm{Td}^{G}(E)$ is an invertible element of $\prod_{i=0}^{\infty} A_{G}^{i}(X)$.

Recall that if $G$ acts freely on a space $Y$ then we have identifications

$$
G^{G}(Y)=G(Y / G) \text { and } C H_{G}^{*}(Y)=C H^{*}(Y / G) .
$$

In what follows, we will use such identifications, often without further comment, and when we compare $\tau^{G}$ and $\tau_{Y / G}$ we are tacitly using these identifications.

We want to define $\tau^{G}: G^{G}(X) \rightarrow \prod_{i=0}^{\infty} C H_{G}^{i}(X)$ so that if $G$ acts freely on $X$, the map $\tau^{G}$ coincides with the non-equivariant map $\tau_{X / G}$. We define $\tau^{G}$ as follows (without assuming the action is free). Choose an embedding of $G$ into a reductive group $G_{1}$ (e.g. $G L_{n}$ ). Suppose $V$ is any representation of $G_{1}$ and $U$ is an open subset of the set of $G_{1}$-stable points of $V$ such that $G$ acts freely on $U$, and $\operatorname{codim}(V-U)>k$ (such pairs always exist by [EG1, Lemma 3]). The action of $G$ on $U$ and hence on $X \times U$ is also free - in particular, proper - so the quotient $X \times^{G} U$ is a separated algebraic space. Define

$$
\rho_{U}: G^{G}(X \times U) \rightarrow C H_{G}^{*}(X \times U)
$$

by

$$
\rho_{U}(\beta)=\frac{\tau_{X \times{ }^{G} U}(\beta)}{\operatorname{Td}^{G}(V)} .
$$

Under the identification $C H_{G}^{*}(X \times U)=C H^{*}\left(X \times{ }^{G} U\right)$, the action of $\mathrm{Td}^{G}(V)$ is that of the Todd class of the vector bundle $X \times{ }^{G}(U \times V) \rightarrow$ $X \times{ }^{G} U$.

If $j<k$ we identify $C H_{G}^{j}(X)$ with $C H_{G}^{j}\left(X \times_{G} U\right)$. If $\alpha \in G^{G}(X)$, we define $\tau^{G}(\alpha) \in \prod_{i=0}^{\infty} C H_{G}^{i}(X)$ to be the element whose $j$-component agrees with that of the image of $\alpha$ under the composition

$$
G^{G}(X) \rightarrow G^{G}(X \times U) \rightarrow C H_{G}^{*}(X \times U),
$$


where the first map is flat pullback.

The reason we define $\tau^{G}$ by first embedding $G$ into a reductive group is that in order to prove that the definition of $\tau^{G}$ is independent of choices, we want to modify our open sets $U$ while still keeping the action proper. If action is proper then the quotient $X \times{ }^{G} U$ is still separated and we can apply non-equivariant Riemann-Roch. If $U$ is contained in the set of stable points for the action of a reductive algebraic group $G_{1}$, then the action of any subgroup of $G_{1}$ is automatically proper.

Proposition 3.1. The definition of $\tau^{G}$ is independent of the choice of $V$ and $U$, and of the embedding of $G$ into a reductive group.

Proof. First fix an embedding of $G$ into a reductive group $G_{1}$. For a given representation $V$, to show that the constructions agree for subsets $U$ and $U^{\prime}$, it suffices to show that the construction using $U$ agrees with that using $U^{\prime \prime}=U \cap U^{\prime}$ (note that since $U \cap U^{\prime}$ is contained in the set of $G_{1}$-stable points, the action of $G_{1}$ on $U \cap U^{\prime}$ is proper). But all constructions in the definition commute with pullback from $X \times U^{\prime \prime}$ to the open set $X \times U$, and from $X \times{ }^{G} U^{\prime \prime}$ to $X \times{ }^{G} U$, so the construction using $U$ agrees with that using $V^{0}$, where $V^{0}$ is the open set of stable points with trivial stabilizer.

To show that the construction is independent of the representation (again keeping $G_{1}$ fixed), it suffices (by symmetry) to show that given $V \supset U$ and $V^{\prime} \supset U^{\prime}$ with $\operatorname{codim}(V-U)$ and $\operatorname{codim}\left(V^{\prime}-U^{\prime}\right)$ greater than $k$, then the construction using $V$ and $U$ agrees with that using $V^{\prime \prime}=V \times V^{\prime}$ and an open subset $U^{\prime \prime}$ of $V^{\prime \prime}$. We can choose the open subset of $V^{\prime \prime}$ arbitrarily, provided the codimension is sufficiently large, so we take the open subset $U^{\prime \prime}=U \times V^{\prime}$. The constructions agree because the following diagram commutes.

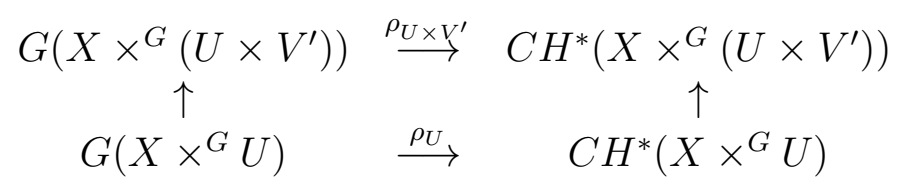

Here the vertical arrows are flat pullback, and commutativity follows from [Fu, Theorem 18.3], using the fact that the relative tangent bundle of the morphism

$$
X \times^{G}\left(U \times V^{\prime}\right) \rightarrow X \times{ }^{G} U
$$

is the bundle

$$
X \times^{G}\left(U \times V^{\prime} \times V^{\prime}\right) \rightarrow X \times^{G}\left(U \times V^{\prime}\right) .
$$

Finally we must show independence of the embedding. Suppose then that $G \rightarrow G_{2}$ is another embedding. Let $V_{i} \supset U_{i}$ be representations 
and open sets for $G_{i}(i=1,2)$. Then $U_{1} \times U_{2}$ is contained in the set of $G_{1} \times G_{2}$-stable points of $V_{1} \times V_{2}$. It suffices to show that the construction made with $\left(V_{1}, U_{1}\right)$ agrees with that made using $\left(V_{1} \times V_{2}, U_{1} \times U_{2}\right)$. This is proved exactly as above, except that in place of the morphism (2), we use

$$
X \times^{G}\left(U_{1} \times U_{2}\right) \rightarrow X \times{ }^{G} U_{1}
$$

Remark 3.1. There is a variant construction of the map $\tau^{G}$, which will be useful below. Let $\mathcal{V} \rightarrow X$ be an equivariant vector bundle such that $G$ acts freely on an open set $\mathcal{U} \subset \mathcal{V}$ which surjects onto $X$. Set

$$
\rho_{\mathcal{U}}(\beta)=\frac{\tau_{\mathcal{U} / G}(\beta)}{\operatorname{Td}^{G} \mathcal{V}}
$$

If $\operatorname{codim}(\mathcal{V}-\mathcal{U})>k$, then the homotopy property of equivariant Chow groups shows that we can identify $C H_{G}^{k}(\mathcal{U})$ with $C H_{G}^{k}(X)$ when $k<j$. Arguing as in the proof of the proposition we can use pairs of the form $(\mathcal{V}, \mathcal{U})$ to obtain an equivalent definition of $\tau^{G}$.

Theorem 3.1. Let $X$ be a separated $G$-space. The equivariant RiemannRoch map

$$
\tau^{G}: G^{G}(X) \rightarrow \prod_{i=0}^{\infty} C H_{G}^{i}(X)
$$

has the following properties.

(a) $\tau^{G}$ factors through the completion map $G^{G}(X) \rightarrow \widehat{G^{G}(X)}$.

(b) $\tau^{G}$ is covariant for proper morphisms.

(c) If $\epsilon \in K^{G}(X)$ and $\alpha \in G^{G}(X)$ then $\tau^{G}(\epsilon \alpha)=\operatorname{ch}^{G}(\epsilon) \tau^{G}(\alpha)$.

(d) Let $f: X \rightarrow Y$ be an equivariant l.c.i. morphism with relative tangent bundle $T_{f}$.

Assume either

(i) $f$ is a quasi-projective morphism (in the sense of [EGA 1], Definition 5.3.1]) or

(ii) $X$ and $Y$ can be equivariantly embedded in smooth schemes, then $\tau^{G}\left(f^{*} \alpha\right)=\operatorname{Td}^{G}\left(T_{f}\right) f^{*} \tau^{G}(\alpha)$.

(e) If $G$ acts freely on $X$, then the map $\tau^{G}$ coincides with the nonequivariant map $\tau_{X / G}$ under the identifications $G^{G}(X)=G(X / G)$ and $C H_{G}^{*}(X)=C H^{*}(X / G)$.

Moreover, $\tau$ is uniquely determined by properties $(d(i))$ and $(e)$.

Proof. Properties (b) and (c) follow from the definition of $\tau^{G}$ and the non-equivariant Riemann-Roch theorem of [Fu, Chapter 18]. Property 
(e) follows because, as in Proposition 3.1, the diagram

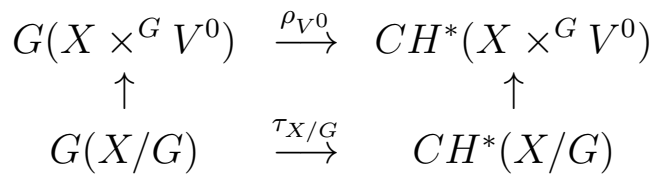

commutes. The non-equivariant Riemann-Roch theorem implies that $\tau_{X / G}$ is an isomorphism after tensoring with $\mathbb{Q}$; hence so is $\tau^{G}$.

If $I_{G}$ is the augmentation ideal of $R(G)$, then $c h^{G}\left(I_{G}^{N}\right) \subset \oplus_{N}^{\infty} R_{G}^{i}$. Thus, by property (c),

$$
\tau^{G}\left(I_{G}^{N} G^{G}(X)\right) \subset \oplus_{N}^{\infty} C H_{G}^{i}(X)
$$

Thus, $\tau^{G}$ restricts to a map

$$
G^{G}(X) / I_{G}^{N} G^{G}(X) \rightarrow \oplus_{i=0}^{N} C H_{G}^{i}(X) .
$$

Taking the limit as $N \rightarrow \infty$ gives the desired factorization

$$
G^{G}(X) \rightarrow \widehat{G^{G}(X)} \rightarrow \prod_{i=0}^{\infty} C H_{G}^{i}(X),
$$

proving (a).

The proof of property (d) uses property (4) of [Fu, Theorem 18.3] but requires some care, particularly in the category of algebraic spaces. Suppose that $f: X \rightarrow Y$ is quasi-projective. Let $U$ be an open set in a representation on which $G$ acts freely. Denote the mixed space $X \times{ }^{G} U$ and $Y \times{ }^{G} U$ by $X_{G}$ and $Y_{G}$. Let $Y^{\prime} \stackrel{p}{\rightarrow} Y$ be a Chow envelope for $Y_{G}$. Then we have a caretsian diagram

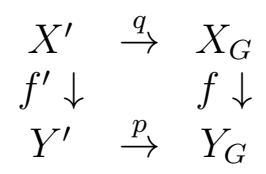

Since $Y^{\prime}$ is a quasi-projective scheme and $f^{\prime}$ is a quasi-projective morphism, follows from [EGA II, Corollary 5.3.3] that $X^{\prime}$ embeds in projective space as well. Hence [Eu, Theorem 18.3(4)] applies to the morphism $f^{\prime}$. If $\alpha \in G\left(Y_{G}\right)$ then $\alpha=p_{*} \alpha^{\prime}$ for some $\alpha^{\prime} \in G\left(Y^{\prime}\right)$. Thus,

$$
\begin{aligned}
\tau\left(f^{*} \alpha\right)=\tau\left(q_{*} f^{\prime} * \alpha^{\prime}\right)=q_{*} \tau\left(f^{\prime} * \alpha\right) & =q_{*}\left[\operatorname{Td}\left(T_{f^{\prime}}\right) f^{\prime} * \tau\left(\alpha^{\prime}\right)\right] \\
& =q_{*}\left[\operatorname{Td}\left(q^{*} T_{f}\right) f^{\prime} * \tau\left(\alpha^{\prime}\right)\right] \\
& =\operatorname{Td}\left(T_{f}\right) f^{*} \tau(\alpha)
\end{aligned}
$$

Taking the limit over all pairs $(V, U)$ in the construction of $\tau^{G}$ gives $(\mathrm{d}(\mathrm{i}))$.

To prove $(\mathrm{d}(\mathrm{ii}))$ argue as follows: Embeds $G \subset G l_{n}$. Replacing $X$ and $Y$ by $X \times G l_{n} / G$ and $Y \times G l_{n}$ we can, by Proposition 3.2 (proved below), assume that $G=G l_{n}$. Suppose that $X \subset M$ and $Y \subset Q$ where 
$M$ and $Q$ are smooth $G$-schemes. Since $G l_{n}$ is special we can chose open sets $U \subset V$ so that $M \times{ }^{G} U$ and $Q \times{ }^{G} U$ are smooth schemes (EG2, Proposition 23]). Thus the mixed space $X_{G}$ and $Y_{G}$ are embedable in smooth schemes so (d(ii)) follows again from [Fu, Theorem 18.3(4)].

Finally, suppose that $\tau^{\prime}$ is another map with properties $(\mathrm{d}(\mathrm{i}))$ and $(\mathrm{e})$. Suppose $\alpha \in G^{G}(X)$ is given and denote by $\tau(\alpha)^{j}$ (resp. $\tau^{\prime}(\alpha)^{j}$ ) the term of $\tau(\alpha)$ (resp. $\left.\tau^{\prime}(\alpha)\right)$ in $C H_{G}^{j}(X)$. Let $(V, U)$ be a representation and open set on which $G$ acts freely such that codim $(V-U)>j$. Let $\pi_{U}: X \times U \rightarrow X \times V \rightarrow X$ be the composition of projection with open inclusion. Since codim $(V-U)>j$ we may identify $C H_{G}^{j}(X)=$ $C H_{G}^{j}(X \times V)=C H_{G}^{j}(X \times U)$. All morphisms are quasi-projective so by property $(\mathrm{d}(\mathrm{i}))$

$$
\tau^{\prime}(\alpha)^{j}=\left(\frac{\tau^{\prime}\left(\pi_{U}^{*} \alpha\right)}{\operatorname{Td}^{G}(V)}\right)^{j} .
$$

Since $G$ acts freely, $\tau^{\prime}$ and $\tau$ coincide on on $X \times U$. Thus

$$
\text { (*) } \quad \tau^{\prime}(\alpha)^{j}=\left(\frac{\tau\left(\pi_{U}^{*} \alpha\right)}{\operatorname{Td}^{G}(V)}\right)^{j}:=\tau(\alpha)^{j} .
$$

Let $E \rightarrow X$ be an equivariant vector bundle on a complete variety, and let $\pi$ denote the morphism $X \rightarrow p t$. Then $\pi_{*}(E)=\sum(-1)^{i}\left[H^{i}(X, E)\right] \in$ $R(G)$. Set $\chi^{G}(E)=\operatorname{ch}\left[\pi_{*}(E)\right] \in \widehat{R(G)}$, and $\operatorname{Td}^{G}(X)=\tau_{X}^{G}\left(\mathcal{O}_{X}\right)$. Applying the general Riemann-Roch theorem to the morphism $X \stackrel{\pi}{\rightarrow} p t$ yields:

Corollary 3.1. (Equivariant Hirzebruch-Riemann-Roch)

$$
\chi^{G}(E)=\pi_{*}\left(\operatorname{ch}^{G}(E) \mathrm{Td}^{G}(X)\right)
$$

in $\widehat{R(G)}$.

3.1. Example: The Weyl character formula. In this section we illustrate the equivariant Riemann-Roch theorem by using it to prove the Weyl character formula for $S L_{2}$. This is essentially a special case of a calculation done by Bott $B$ using the Atiyah-Singer index theorem. This proof is different than some other proofs in that it does not use a localization theorem to reduce to a computation at the fixed point locus of the action of a maximal torus on the flag variety.

Let $T=\mathbb{G}_{m}$ acting on $\mathbf{P}^{1}$ with weights $1,-1$. What we will calculate is $\chi^{T}\left(\mathcal{O}_{\mathbf{P}^{1}}(n)\right) \in \prod_{i=0}^{\infty} C H_{T}^{i}(p t)$. This calculation is related to representations of $S L_{2}$, since we can view $T$ as embedded in $S L_{2}$ as a maximal torus. Then $\sum(-1)^{i} H^{i}\left(\mathbf{P}^{1}, \mathcal{O}_{\mathbf{P}^{1}}(n)\right)$ is a virtual representation of $S L_{2}$ and $\chi^{T}\left(\mathcal{O}_{\mathbf{P}^{1}}(n)\right)$ gives a formula for the restriction of 
this representation to $T$. For $n \geq 0$ the higher cohomology groups of $\left.\mathcal{O}_{\mathbf{P}^{1}}(n)\right)$ vanish, the resulting representation of $S L_{2}$ is irreducible, and our formula for $\chi^{T}\left(\mathcal{O}_{\mathbf{P}^{1}}(n)\right)$ gives the Weyl character formula.

Here is the calculation. We have $A_{T}^{*}(p t)=\mathbb{Q}[t]$, and

$$
\prod_{i=0}^{\infty} A_{G}^{i}(X) \cong \prod_{i=0}^{\infty} C H_{T}^{i}(p t)=\mathbb{Q}[[t]] .
$$

The Chern character $c h^{T}: R_{T} \rightarrow \prod_{i=0}^{\infty} A_{G}^{i}(X)$ is given explicitly as follows. If $V$ is a representation of $T$, write

$$
V=k_{n_{1}} \oplus \cdots \oplus k_{n_{r}},
$$

where $k_{n_{i}}$ is the 1-dimensional representation of $T$ with weight $n_{i}$ (the weights $n_{i}$ need not be distinct). Then $\operatorname{ch}^{T}(V)=\sum e^{n_{i} t}$.

In [EG2, Section 3.3] we computed $A_{T}^{*}\left(\mathbf{P}^{1}\right) \simeq \mathbb{Q}[t, h] /(t+h)(t-h)$ when $T$ acts with weights \pm 1 . Under this identification, $c_{1}^{T}\left(\mathcal{O}_{\mathbf{P}^{1}}(n)\right)=$ $n h$ and $c_{1}^{T}\left(T_{\mathbf{P}^{1}}\right)=2 h$. Thus

$$
\pi_{*}\left(\operatorname{ch}^{T}\left(\mathcal{O}_{\mathbf{P}^{1}}(n)\right) \operatorname{Td}^{T}\left(\mathbf{P}^{1}\right)\right)=\pi_{*}\left(e^{n h} \frac{2 h}{1-e^{-2 h}}\right) .
$$

Lemma 3.1. If $p(h) \in C H_{T}^{*}\left(\mathbf{P}^{1}\right) \simeq \mathbb{Q}[t, h] /(t+h)(t-h)$, then $\pi_{*}(p(h))$ is given by

$$
\pi_{*}(p(h))=\frac{p(t)-p(-t)}{2 t} .
$$

The same formula holds for $\pi_{*}: \prod_{i=0}^{\infty} C H_{T}^{i}\left(\mathbf{P}^{1}\right) \rightarrow \prod_{i=0}^{\infty} C H_{T}^{i}(p t)$.

Proof. If $B T$ is a model for calculating $C H_{T}^{*}$, then the model $\mathbf{P}_{T}^{1}$ for calculating $C H_{T}^{*}\left(\mathbf{P}^{1}\right)$ is a $\mathbf{P}^{1}$ bundle over $B T$. The class of a section is $h$, so $\pi_{*}\left(\sum a_{i} t^{i}+h \sum b_{j} t^{j}\right)=\sum b_{j} t^{j}$. Now if $p(h)=\sum a_{i} h^{i} \in$ $C H_{T}^{*}\left(\mathbf{P}^{1}\right)$, then we can write (using the relation $\left.h^{2}=t^{2}\right) x=\sum a_{2 i} t^{2 i}+$ $h \sum a_{2 i+1} t^{2 i}$, so

$$
\pi_{*}(p(h))=\sum a_{2 i+1} t^{2 i}=\frac{p(t)-p(-t)}{2 t}
$$

which is the desired formula. The same argument works for the completed Chow groups.

The lemma implies that $\chi^{T}\left(\mathcal{O}_{\mathbf{P}^{1}}(n)\right)$ is equal to

$$
\pi_{*}\left(e^{n h} \frac{2 h}{1-e^{-2 h}}\right)=\pi_{*}\left(2 h \frac{e^{(n+1) h}}{e^{h}-e^{-h}}\right)=\frac{e^{(n+1) t}-e^{-(n+1) t}}{e^{t}-e^{-t}}=\sum_{i=0}^{n} e^{(n-2 i) t},
$$

which is the desired formula. 
3.2. Change of groups. Let $H \subset G$ be an algebraic subgroup. In this section we discuss the relationship between $G$-equivariant and $H$ equivariant groups, and the corresponding Riemann-Roch maps $\tau^{G}$ and $\tau^{H}$.

Lemma 3.2. Given an action of $G \times H$ on $X$, with $H=1 \times H$ acting freely, there is a commutative diagram

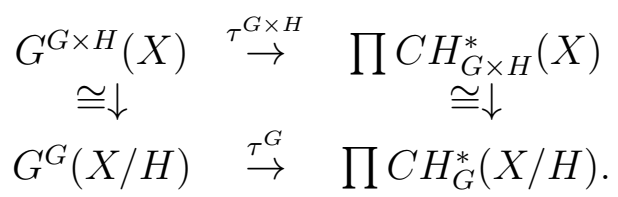

Proof. Let $V$ be a representation of $G \times H$ and let $U$ be an open set on which $G$ acts freely. Then $\mathcal{V}=X \times{ }^{H} V$ is a $G$-equivariant vector bundle on $X / H$. The group $G$ acts freely on the open set $\mathcal{U}=X \times{ }^{H} U$ which surjects onto $X$. Identifying $\mathcal{U} / G$ with $X \times{ }^{G \times H} U$ we see that the maps $\rho_{U}$ and $\rho_{\mathcal{U}}$ are the same. Using pairs of the form $(V, U)$ we define $\tau^{G \times H}$ as $\rho_{U}$, as before. On the other hand, by Remark 3.1 we can use vector bundles and open sets of the form $(\mathcal{V}, \mathcal{U})=\left(X \times{ }^{H} V, X \times{ }^{H} U\right)$ to define $\tau_{X / H}^{G}$ as $\rho_{\mathcal{U}}$. Hence the maps coincide.

In $K$-theory, part (a) of the next proposition is due to Thomason [Tho3], following ideas that go back to Atiyah and Segal [AS]. Part (c) has apparently been used implicitly by Thomason ([Tho4]), but we do not know of an explicit statement or proof.

Proposition 3.2. Let $H$ be a subgroup of $G$, and let $X$ be an $H$-space.

(a) There is a natural isomorphism of $R(G)$-modules $G^{G}\left(G \times{ }^{H} X\right) \simeq$ $G^{H}(X)$, and a natural isomorphism of $A_{G}^{*}$-modules $\oplus C H_{G}^{i}\left(G \times{ }^{H} X\right) \simeq$ $\oplus C H_{H}^{i}(X)$.

(b) The isomorphisms are compatible with the $\tau$ maps, i.e., the following diagram commutes:

$$
\begin{array}{ccc}
G^{G}\left(G \times{ }^{H} X\right) & \stackrel{\tau^{G}}{\rightarrow} & \prod C H_{G}^{*}\left(G \times{ }^{H} X\right) \\
\cong \downarrow & & \cong \downarrow \\
G^{H}(X) & \stackrel{\tau^{H}}{\rightarrow} & \prod C H_{H}^{*}(X) .
\end{array}
$$

(c) If the $H$-action on $X$ is the restriction of a G-action, then $G \times{ }^{H} X \cong G / H \times X$. Under the isomorphism in (a), the "forgetful" map $G^{G}(X) \rightarrow G^{H}(X)$ corresponds to the flat pullback $G^{G}(X) \rightarrow$ $G^{G}(G / H \times X)$. A similar statement holds for Chow groups.

We will only prove parts (a) and (c) for Grothendieck groups; the arguments for Chow groups are similar. Before proving the proposition, we recall some general facts about equivariant sheaves. 
Let $G$ be an algebraic group and let $X$ be a $G$-space, with $\pi, a$ : $G \times X \rightarrow X$ the projection and action maps (taking $(g, x)$ to $x$ and $g x$ respectively). A coherent sheaf $\mathcal{F}$ on $X$ is $G$-equivariant if there is an isomorphism $\theta: \pi^{*} \mathcal{F} \rightarrow a^{*} \mathcal{F}$ satisfying a cocycle condition coming from the group law. (For a precise description of the cocycle condition, see [Tho3, Section 1.2].) If $G_{1} \times G_{2}$ acts on $X$, it is convenient to think of a $G_{1} \times G_{2}$-equivariant sheaf on $X$ as a triple $\left(\mathcal{F}, \theta_{1}, \theta_{2}\right)$, where $\theta_{i}$ plays the role of $\theta$ for $G_{i}$, and the $\theta_{i}$ also satisfy a condition coming from the fact that the $G_{1}$ action commutes with the $G_{2}$-action.

Now let $G$ and $H$ be algebraic groups and let $f: X \rightarrow Y$ be a map of spaces. Consider the following three constructions of equivariant sheaves.

First, suppose $X$ and $Y$ are $H$-spaces and $f$ is an $H$-equivariant map. If $\mathcal{G}$ is an $H$-equivariant sheaf on $Y$, then $f^{*} \mathcal{G}$ is an $H$-equivariant sheaf on $X$. The isomorphism $\pi^{*}\left(f^{*} \mathcal{G}\right) \stackrel{E^{f}}{\rightarrow} a^{*}\left(f^{*} \mathcal{G}\right)$ is defined by $E^{f}=$ $(1 \times f)^{*} \theta$ (the notation $E^{f}$ is used to indicate equivariance), where $\theta$ gives the equivariant structure on $H$, and $1 \times f: H \times X \rightarrow H \times Y$ takes $(h, x)$ to $(h, f(x))$.

Second, suppose $X$ is a $G$-space and $f$ is a invariant map, i.e., $f(g x)=f(x)$ for all $g$ and $z$. If $\mathcal{G}$ is any sheaf on $Y$ then the sheaf $f^{*} \mathcal{G}$ acquires a $G$-action. We denote the isomorphism $\pi^{*}\left(f^{*} \mathcal{G}\right) \rightarrow a^{*}\left(f^{*} \mathcal{G}\right)$ by $I^{f}$ (the $I$ stands for invariant); it is the canonical isomorphism coming from the identity $f \circ \pi=f \circ a$. (This can be thought of the special case of an equivariant map where $G$ acts trivially on $Y$ and on $\mathcal{G}$, but we prefer to distinguish the cases.) If $f: X \rightarrow Y$ is a principal bundle quotient (i.e., a torsor) then there is an equivalence of categories between $G$-equivariant coherent sheaves on $X$ and coherent sheaves on $Y$ Tho3. The reason is that in this case, $G \times X \simeq X \times_{Y} X$, and the equivariant structure $\theta$ is the same as descent data for the morphism $X \rightarrow Y$.

Third, if the map $f$ is both $H$-equivariant and $G$-invariant, then (writing $H=G_{1}, G=G_{2}$ ) there is an equivalence of categories between $G_{1} \times G_{2}$-equivariant sheaves on $X$ and $H$-equivariant sheaves on $Y$. The $H$-equivariant sheaf $\mathcal{G}$ on $Y$ corresponds to the $G_{1} \times G_{2}$-equivariant sheaf $\left(f^{*} \mathcal{F}, E_{1}^{f}, I_{2}^{f}\right)$ on $X$. (The condition that the $G_{1}$-action commute with the $G_{2}$-action can be seen by viewing $f$ as a $G_{1} \times G_{2}$-equivariant map, where $G_{2}$ acts trivially on the target. This uses the observation that invariant maps are a special case of equivariant maps.)

Proof of Proposition 3.2. Let $X$ be an $H$-space. Write $G_{1}=H$ and $G_{2}=G$, and define an $G_{1} \times G_{2}$-action on $G \times X$ by:

$$
g_{1} \cdot(g, x)=\left(g g_{1}^{-1}, g_{1} x\right)
$$




$$
g_{2} \cdot(g, x)=\left(g_{2} g, x\right)
$$

The projection $\pi: G \times X \rightarrow X$ is $H$-equivariant and $G$-invariant, and the projection $G \times X \rightarrow G \times{ }^{H} X$ is $G$-equivariant and $H$-equivariant. Hence we have equivalences of categories between:

$H$-equivariant coherent sheaves on $X$,

$G \times H$-equivariant coherent sheaves on $G \times X$, and

$G$-equivariant coherent sheaves on $G \times{ }^{H} X$.

Hence $G^{G}\left(G \times{ }^{H} X\right) \simeq G^{H}(X)$. Under these equivalences of categories, the $G$-equivariant vector bundle $V \times\left(G \times{ }^{H} X\right) \rightarrow G \times{ }^{H} X$ corresponds to the $H$-equivariant vector bundle $V \times X \rightarrow X$. This translates into the fact that the isomorphism is an isomorphism of $R(G)$-modules. This proves (a) in $K$-theory. Part (b) follows from Lemma 3.2.

We prove (c). Here let $G_{1}=G_{2}=G$ and write $G \times G=G_{1} \times G_{2}$. Define a $G_{1} \times G_{2}$-action on $G \times X$ by the same formulas as above. Given a $G$-equivariant sheaf $\mathcal{F}$ on $X$, we can define a $G_{1} \times G_{2}$-equivariant sheaf on $G \times X$ in 2 ways:

First, the map $\pi: G \times X \rightarrow X$ is equivariant with respect to the $G_{1}$ action $\left(G_{1}=G\right.$ acts as usual on $\left.X\right)$ and invariant with respect to the $G_{2}$-action. Hence this gives a $G_{1} \times G_{2^{-}}$equivariant sheaf $\left(\pi^{*} \mathcal{F}, E_{1}^{\pi}, I_{2}^{\pi}\right)$ on $G \times X$.

Second, the map $a: G \times X \rightarrow X$ is invariant for the $G_{1}$ action and equivariant for the $G_{2}$-action. This gives a $G_{1} \times G_{2}$-equivariant sheaf $\left(a^{*} \mathcal{F}, I_{1}^{a}, E_{2}^{a}\right)$ on $G \times X$.

The fact that $\theta: \pi^{*} \mathcal{F} \rightarrow a^{*} \mathcal{F}$ satisfies the cocycle condition implies:

Lemma 3.3. The map $\theta: \pi^{*} X \rightarrow a^{*} X$ is an isomorphism of $G_{1} \times G_{2}$ equivariant sheaves.

A consequence of the lemma is:

Proposition 3.3. The pullback $\pi^{*}$ (resp. $a^{*}$ ) gives an equivalence of categories between $G_{1}$ (resp. $G_{2}$ ) equivariant sheaves on $X$ and $G_{1} \times$ $G_{2}$-equivariant sheaves on $G \times X$, such that (identifying $G_{1}$ and $G_{2}$ with $G$ ) the composition $\left(a^{*}\right)^{-1} \circ \pi^{*}$ is the identity. Hence there are isomorphisms of Grothendieck groups

$$
G^{G_{1}}(X) \stackrel{\pi^{*}}{\rightarrow} G^{G_{1} \times G_{2}}(G \times X) \stackrel{\left(a^{*}\right)^{-1}}{\rightarrow} G^{G_{2}}(X)
$$

such that $\left(a^{*}\right)^{-1} \circ \pi^{*}$ is the identity.

Proof. $\pi^{*}$ and $a^{*}$ are equivalences of categories because $\pi$ and $a$ are principal bundle quotients. By the lemma, if $\mathcal{F}$ is any $G$-equivariant sheaf on $X$, then $\pi^{*} \mathcal{F}$ and $a^{*} \mathcal{F}$ are isomorphic $G_{1} \times G_{2}$-equivariant sheaves on $G \times X$. Hence $\left(a^{*}\right)^{-1} \circ \pi$ is the identity. 
Now view $H$ as a subgroup of $G_{1}$. We have $G_{2}$-equivariant maps

$$
G \times X \stackrel{q}{\rightarrow}(G \times X) / H \stackrel{\bar{a}}{\rightarrow} X
$$

where $q$ is the quotient map and $\bar{a}$ is induced from $a$. Identify $(G \times$ $X) / H$ with $G / H \times X$ by the isomorphism $(g, x) H \mapsto(g H, g x)$. Under this identification, the $G_{1}$-action on $(G \times X) / H$ becomes the product action on $G / H \times X$, and

$$
q(g, x)=(g H, g x)
$$

Hence $\bar{a}$ is the second projection:

$$
\bar{a}(g H, x)=x \text {. }
$$

The map $q: G \times X \rightarrow X$ is an $H \times 1$ torsor, and the map $\pi: G \times X \rightarrow X$ is a $1 \times G_{2}$-torsor. Hence

$$
G^{G_{2}}(G / H \times X) \stackrel{q^{*}}{\rightarrow} G^{H \times G_{2}}(G \times X)
$$

and

$$
G^{H}(X) \stackrel{\pi^{*}}{\rightarrow} G^{H \times G_{2}}(G \times X)
$$

are isomorphisms. The composition $q^{*} \circ\left(a^{*}\right)^{-1}$ gives an isomorphism $G^{G}(G / H \times X) \rightarrow G^{H}(X)$.

To prove that this isomorphism corresponds to flat pullback, consider the following diagram, where the vertical arrows are the forgetful maps:

$$
\begin{array}{ccccc}
G^{G_{2}}(X) & \stackrel{\bar{a}^{*}}{\rightarrow} G^{G_{2}}(G / H \times X) \stackrel{q^{*}}{\rightarrow} & G^{H \times G_{2}}(G \times X) & \stackrel{\left(\pi^{*}\right)^{-1}}{\rightarrow} & G^{H}(X) \\
= & \stackrel{a^{*}}{\longrightarrow} & G^{G_{1} \times G_{2}}(G \times X) & \stackrel{\left(\pi^{*}\right)^{-1}}{\rightarrow} & G^{G_{2}}(X) \\
G^{G_{2}}(X) & & &
\end{array}
$$

The diagram commutes by the definitions of the equivariant structures. By Proposition 3.3 composition along the bottom row is the identity. Consider the two compositions (identifying $G_{2}$ with $\left.G\right) G^{G}(X) \rightarrow$ $G^{H}(X)$. By considering the composition along the bottom row, we see this is the forgetful map. On the other hand, along the top row, $G^{H}(X)$ is identified with $G^{G}(G / H \times X)$. Under this identification, the map $G^{G}(X) \rightarrow G^{H}(X)$ becomes the flat pullback $\bar{a}^{*}$; as noted above, $\bar{a}$ is the second projection. This proves the result.

\section{The Riemann-Roch isomorphism}

All spaces considered here are again assumed to be separated.

Let $G \subset G L_{n}$ and let $I \subset R\left(G L_{n}\right)$ be the augmentation ideal. In this section we will use the notation $\widehat{G^{G}(X)}$ to denote the $I$-adic completion of $G^{G}(X) \otimes \mathbb{Q}$, and continue to denote $C H_{G}^{i}(X) \otimes \mathbb{Q}$ by $C H_{G}^{i}(X)$.

The purpose of this section is to prove the following theorem. 
Theorem 4.1. Let $X$ be a separated algebraic space. The map

$$
\tau^{G}: \widehat{G^{G}(X)} \rightarrow \prod_{i=0}^{\infty} C H_{G}^{i}(X) \otimes \mathbb{Q}
$$

an isomorphism.

Remark 4.1. By Corollary $\widehat{6.1} \widehat{G^{G}(X)}$ is also the completion of $G^{G}(X)$ with respect to $I_{G} \subset R(G)$.

If $G \subset G L_{n}$, then we obtain by Proposition 3.2 a commutative diagram

$$
\begin{aligned}
G^{G L_{n}}\left(\widehat{G L_{n} / G} \times X\right) & \cong c \\
\downarrow \tau^{G L_{n}} & \widehat{G^{G}(X)} \\
\prod_{i=1}^{\infty} C H_{G L_{n}}^{i}\left(G L_{n} / G \times X\right) & \cong \prod_{i=1}^{\infty} C \tau_{G}^{i}(X)
\end{aligned}
$$

Thus, to prove the theorem it suffices to show $\tau^{G L_{n}}$ is an isomorphism. We will do this in two steps.

4.1. $G=B$ is the group of upper triangular matrices. Let $\mathcal{V}=\left\{V_{n}, U_{n}\right\}$ be the system of good representations and open subsets constructed in the proof of Theorem 2.2. Since $G$ acts freely on $X \times U_{n}$, Proposition 3.1.(b) implies that

$$
\tau^{G}: G^{G}\left(X \times U_{n}\right) \rightarrow C H_{G}^{*}\left(X \times U_{n}\right)
$$

is an isomorphism.

By Theorem 3.1, the map $\tau^{G}$ is compatible with the maps in the inverse system of representations constructed in Section 2. In this way we obtain an isomorphism

$$
\tau^{G}: \lim _{\leftarrow(V, U) \in \mathcal{V}}\left(G^{G}(X) / \operatorname{ker} k_{n}\right) \rightarrow \lim _{\leftarrow(V, U) \in \mathcal{V}} C H_{G}^{*}(X) / \operatorname{ker} r_{n} .
$$

By Proposition 2.2, the $I_{B^{-}}$adic completion of $G^{B}(X)$ is isomorphic to the $I$-adic completion $\widehat{G^{B}(X)}$, so by Theorem 2.1,

$$
\lim _{\leftarrow(V, U) \in \mathcal{V}}\left(G^{B}(X) / \operatorname{ker} k_{n}\right) \simeq \widehat{G^{B}(X)} .
$$

Also, by Theorem 2.1 and Proposition 2.1,

$$
\lim _{\leftarrow(V, U) \in \mathcal{V}} C H_{B}^{*}(X) / \operatorname{ker} r_{n} \simeq \widehat{C H_{B}^{*}(X)} \simeq \prod_{i=0}^{\infty} C H_{B}^{i}(X) .
$$

The case $G=B$ follows. 
4.2. $G=G L_{n}$. Let $\pi: G / B \times X \rightarrow X$ be projection to the second factor. By Proposition 3.2, we identify $G^{B}(X)$ with $G^{G}(X \times G / B)$ so that the forgetful map $i_{!}: G^{G}(X) \rightarrow G^{B}(X)$ corresponds to flat pullback $\pi^{*}: G^{G}(X) \rightarrow G^{G}(X \times G / B)$.

Since $B$ is the group of upper triangular matrices, the quotient $G / B$ is complete and we can define a map $i_{!}: G^{B}(X) \rightarrow G^{G}(X)$ by using the identification $G^{B}(X) \cong G^{G}(G / B \times X)$ and setting $i_{\text {! }}$ to be the proper push-forward $\pi_{!}: G^{G}(G / B \times X) \rightarrow G^{G}(X)$.

Lemma 4.1. The composition $i_{!} i^{!}=i d$, so we can identify $G^{G}(X)$ as a summand in $G^{B}(X)$.

Proof. This follows from the argument of [AS], using the fact that $G / B$ is a tower of projective bundles and the projective bundle theorem of [Tho3, Theorem 3.1].

We now turn to the Chow theory. Using the identification $C H_{B}^{*}(X) \simeq$ $C H_{G}^{*}(G / B \times X)$ to define a map

$$
i^{*}: \prod_{0}^{\infty} C H_{G}^{i}(X) \rightarrow \prod_{i=0}^{\infty} C H_{B}^{i}(X) \simeq \prod_{i=0}^{\infty} C H_{G}^{i}(G / B \times X)
$$

by the formula

$$
\alpha \mapsto \mathrm{Td}^{G}\left(T_{G / B}\right) \pi^{*}(\alpha)
$$

We also set

$$
i_{*}: C H_{B}^{*}(X) \rightarrow C H_{G}^{*}(X)
$$

to be the composition

$$
C H_{B}^{*}(X) \simeq C H_{G}^{*}(G / B \times X) \stackrel{\pi_{*}}{\rightarrow} C H_{G}^{*}(X) .
$$

Lemma 4.2. The composition $i_{*} i^{*}=i d$, so we can identify $C H_{G}^{*}(X)$ as a summand in $C H_{B}^{*}(X)$.

Proof. In general, if $f: M \rightarrow N$ is a smooth proper morphism with $f_{!}\left[\mathcal{O}_{M}\right]=\left[\mathcal{O}_{N}\right]$, then the Riemann-Roch theorem implies that

$f_{*}\left(\operatorname{Td}\left(T_{f}\right) f^{*} \beta\right)=\beta$ for all $\beta \in C H^{*}(N)$. (Proof: Write $\beta=\tau_{N} \alpha, \alpha \in$ $G(Y)$, and calculate $f_{*} \tau_{M} f^{!} \alpha$ in two ways. First, it equals $\tau_{N} f_{!} f^{!} \alpha=$ $\tau_{N} \alpha$ since $f_{!}\left[\mathcal{O}_{M}\right]=\left[\mathcal{O}_{N}\right]$ implies $f_{!} f^{!} \alpha=\alpha$. On the other hand, it equals $f_{*}\left(\operatorname{Td}\left(T_{f}\right) f^{*}\left(\tau_{N} \alpha\right)\right.$ by Riemann-Roch.) Applying this to the case where $M$ and $N$ are the mixed spaces $(G / B \times X)^{G} U$ and $X \times{ }^{G} U$ yields the result.

Lemma 4.3. We have:

(i) $i_{!} \tau^{G}=\tau^{B} i_{*}$

(ii) $\tau^{B} i^{!}=i^{*} \tau^{G}$. 
Proof. This follows from the previous lemmas and the property $(\mathrm{d}(\mathrm{i}))$ of Theorem 3.1.

Since $\tau_{B}$ is an isomorphism the previous lemmas imply $\tau_{G}$ is also an isomorphism when $G=G L_{n}$. This proves the theorem.

\section{ACtions With Finite STABILIZERS}

Throughout this section we assume that the group $G$ acts on the space $X$ with finite stabilizers. We will also assume that all groups are taken over $\mathbb{Q}$.

In this situation, we can obtain more refined information about the completions in Section 2. In particular we prove the following theorem.

Theorem 5.1. Suppose that an algebraic group $G$ on a space $X$ with finite stabilizers. Let $I_{G}$ be the augmentation ideal. Then there is an isomorphism

$$
\widehat{G^{G}(X)} \rightarrow G^{G}(X)_{I}
$$

We begin with a commutative algebra lemma.

Lemma 5.1. Let $R$ be a ring and $I$ a maximal ideal and let $M$ be an $R$-module. Suppose that $I^{k} M_{I}=0$. Then $M_{I}=\widehat{M}$ where $\widehat{\text { denotes the }}$ I-adic completion.

Proof. The rings $R / I^{k}$ are local, so $\left(R / I^{k}\right)_{I}=R / I^{k}$. Thus,

$$
M_{I} / I^{k} M_{I}:=M \otimes\left(R_{I} / I^{k} R_{I}\right)=M \otimes R / I^{k}:=M / I^{k} M .
$$

Hence $\widehat{M}_{I} \cong \widehat{M}$. Since $I^{k} M_{I}=0, M_{I}$ is $I$-adically complete, proving the result.

Because of the lemma, Theorem 5.1 is an immediate consequence of the following result, whose proof uses Corollary 6.2.

Proposition 5.1. If $G$ on $X$ with finite stabilizers then for sufficiently large $k$,

$$
I^{k} G^{G}(X)_{I}=0
$$

Proof. The proof proceeds as in previous proofs, by building up from a torus to $G L_{n}$ to a general group.

Step 1. $G=T$ is a torus. By Thomason's generic slice theorem, there is a $T$-equivariant open subset $U \subset X$ such that $U$ is equivariantly isomorphic to $S \times Y$ where $S=T / H$ for a diagonalizable subgroup $H \subset T$, and $T$ acting trivially on the $Y$. If $W$ is any representation of $T$ which is trivial on $H$, then the vector bundle $U \times W \rightarrow U$ is equivariantly trivial, so $([W]-\operatorname{dim} W) G^{T}(U)=0$. Hence, under the 
map $R(S) \rightarrow R(T)$, the ideal $J=I_{S} R(T)$ annihilates $G^{T}(U)$. Note that $J \subset I_{T}$.

Because $H$ is finite, we can choose a basis $e_{1}, \ldots, e_{n}$ of $\widehat{T}$ such that $d_{1} e_{1}, \ldots, d_{n} e_{n}$ is a basis for $\widehat{S}$, where the $d_{i}$ are positive integers. Using this basis, we have

$$
R(T) \cong \mathbb{Q}[\widehat{T}] \cong \mathbb{Q}\left[t_{1}, \ldots, t_{n}\right]
$$

and

$$
J=\left(t_{1}^{d_{1}}-1, \ldots, t_{n}^{d_{n}}-1\right) \subset I_{T}=\left(t_{1}-1, \ldots, t_{n}-1\right) .
$$

Hence $J_{I_{T}}=I_{T} R(T)_{I_{T}}$, so $I_{T} G^{T}(U)_{I_{T}}=0$.

By Noetherian induction we may assume that $I^{l} G^{T}(X-U)_{I}=0$ for some sufficiently large $k$. Then by the localization exact sequence, $I^{k+1} G^{T}(X)_{I}=0$.

Step 2. $G=G L_{n}$. Let $B$ be the group of upper triangular matrices in $G L_{n}$ and let $T$ be the group of diagonal matrices. Restriction from $B$ to $T$ induces isomorphisms $R(B) \cong R(T)$ and $G^{B}(X) \cong G^{T}(X)$ (see Tho4, Proof of Theorem 1.11]). Claim: There is an ideal $J_{G} \subset I_{G}$ with the support of $R(G) / J_{G}$ finite, such that $J_{G} G^{G}(X)=0$. For, $R(G) \cong R(B)^{W} \hookrightarrow R(B)$ is finite, so if $J_{G}$ is the inverse image of $J_{B}:=J_{T}$ under this map, then the support of $R(G) / J_{G}$ is finite. Since $G^{G}(X)$ is an $R(G)$-submodule of $G^{B}(X)$ (see the proof of Theorem 4.1), we have $J_{G} G^{G}(X)=0$, proving the claim.

Because $R(G) / J_{G}$ has finite support and $J_{G}$ is contained in the maximal ideal $I_{G}$, it follows that $J_{G} R(G)_{I_{G}}$ is $I_{G}$-primary, hence contains $I_{G}^{k} R(G)_{I_{G}}$ for some $k$ (note that $R\left(G=G l_{n}\right)=\mathbb{Z}\left[t_{1}, \ldots, t_{n}\right]^{S_{n}}$ is Noetherian). Then $I_{G}^{k} G^{G}(X)_{I_{G}}=0$ as desired.

Step 3. The general case. Embed $G \subset G L_{n}$. In this case, $G^{G}(X)=$ $G^{G L_{n}}\left(G L_{n} / G \times X\right)$. Hence there exists an ideal $J_{G L_{n}} \subset I_{G L_{n}}$ with support of $R\left(G L_{n}\right) / J_{G L_{n}}$ finite, such that $J_{G L_{n}} G^{G}(X)=0$. Let $\mathfrak{q}$ be the $I_{G L_{n}}$-primary component of $J_{G L_{n}}$; then $\mathfrak{q} \supset I_{G L_{n}}^{k}$ for some $k$. Hence $I_{G L_{n}}^{k} G^{G}(X)_{I_{G L_{n}}}=0$, or equivalently, given $x \in G^{G}(X)$ and $a \in I_{G L_{n}}^{k}$, there exists $b \in R(G)-I_{G L_{n}}$ such that $a b x=0$.

Now, the action of $R\left(G L_{n}\right)$ on $G^{G}(X)$ factors through $\phi: R\left(G L_{n}\right) \rightarrow$ $R(G)$. Also, the augmentation map $R\left(G L_{n}\right) \rightarrow \mathbb{Q}$ factors through $\phi$, so if $x \in R\left(G L_{n}\right)-I_{G L_{n}}$ then $\phi(x) \in R(G)-I_{G}$. Corollary 6.2 implies that for some $d, I_{G}^{d} \subset \phi\left(I_{G L_{n}}\right) R(G)$. This implies that $I_{G}^{d k} G^{G}(X)_{I_{G}}=0$. Indeed, if $a^{\prime} \in I_{G}^{d k}$ and $x \in G^{G}(X)$, write $a^{\prime}=\phi(a)$ for $a \in I_{G L_{n}}^{k}$, choose $b$ as in the preceding paragraph; then $b^{\prime}=\phi(b) \in R(G)-I(G)$ and $a^{\prime} b^{\prime} x=0$. This proves the result.

Remark 5.1. The proof of the proposition implies that for $G=T$ or $G=G L_{n}$, that there is an ideal $J \subset I_{G} \subset R(G)$ with the support of 
$R(G) / J$ finite, such that $J G^{T}(X)=0$. We will use this fact without further comment in the proof Theorem 5.2. In characteristic 0, the fact holds for arbitrary $G$, since by Segal, Proposition 3.2] the map $R\left(G L_{n}\right) \rightarrow R(G)$ is finite. However, in characteristic $p$ we don't even know if $R(G)$ is always Noetherian!

If $G$ acts with finite stabilizers then the rational equivariant Chow groups $C H_{G}^{*}(X)_{\mathbb{Q}}$ are generated by invariant cycles on $X$ [EG2]. In particular $C H_{G}^{i}(X)_{\mathbb{Q}}=0$ for and $i>\operatorname{dim} X$, so the equivariant Chow groups are complete.

Thus the Riemann-Roch theorem can be restated.

Corollary 5.1. Suppose that $X$ is separated and $G$ acts with finite stabilizers. There is a map

$$
\tau: G^{G}(X) \rightarrow G^{G}(X)_{I} \stackrel{\sim}{\rightarrow} C H_{G}^{*}(X)_{\mathbb{Q}}
$$

satisfying properties (a)-(e) of Theorem 3.1.

When $G$ acts on $X$ with finite reduced stabilizers then Vistoli $V \mathbb{1}$ stated a theorem which asserted the existence of a map

$$
\tau_{X}: G^{G}(X) \otimes \mathbb{Q} \rightarrow C H^{*}([X / G] \otimes \mathbb{Q})
$$

where here $[X / G]$ is the Deligne-Mumford quotient stack. By EG2, Proposition 14],

$$
C H^{*}([X / G]) \otimes \mathbb{Q}=C H_{G}^{*}(X) \otimes \mathbb{Q} .
$$

It easy to check that his map satisfies properties (d) and (e) of Theorem 3.1. Thus by Theorem 3.1 Vistoli's $\tau_{X}$ is the same as ours. Vistoli noted that his map need not be an isomorphism and made a conjecture about its kernel.

Vistoli's conjecture is true and the statement can be refined.

Corollary 5.2. (Vistoli's conjecture)

$$
\alpha \in \operatorname{ker}\left(\tau_{X}^{G}: G^{G}(X) \rightarrow C H^{*}([X / G])_{\mathbb{Q}}\right)
$$

if and only if $\epsilon \alpha=0$ where $\epsilon \in R(G)$ is a virtual representation of non-zero rank.

Proof. The kernel is just the kernel of the localization map $G^{G}(X) \rightarrow$ $G^{G}(X)_{I}$. 
5.1. The case $G$ is diagonalizable. Let $P=P_{0}, P_{1}, \ldots P_{k}$ be the prime ideals in the support of $G_{i}^{G}(X)$ as an $R(G)$-module. Following Segal Segal each prime $P_{i}$ corresponds to a finite subgroup (called the support of $\left.P_{i}\right) H_{i} \subset G$. It is defined as the minimal element of the set of subgroups $H \subset G$ such that $P_{i} \in \operatorname{Im}(\operatorname{Spec} R(H) \rightarrow \operatorname{Spec} R(G))$. Note that different $P_{i}$ 's may have the same support. This group is a priori only defined up to conjugation as a subgroup of $G$. However, since $G$ is abelian, the $H_{i}$ 's are uniquely determined. (See [Tho5, Proposition 1.2] for how this works in characteristic $p$.)

Denote by $X^{i}$ the subscheme fixed by $H_{i}$.

Theorem 5.2. Suppose that $G$ is diagonalizable and acts on a separated space $X$ with finite stabilizers. Then

$$
\left(G^{G}(X)_{P_{i}} \simeq C H_{G / H_{i}}^{*}\left(X^{i}\right) \otimes_{R\left(G / H_{i}\right)} R(G)_{P_{i}} .\right.
$$

In particular, there is an isomorphism

$$
G^{G}(X) \simeq \prod_{i} C H_{G / H_{i}}^{*}\left(X^{i}\right) \otimes_{R\left(G / H_{i}\right)} R(G)_{P_{i}}
$$

Remark 5.2. This result was first obtained by Angelo Vistoli (private communication). It is also related to the Riemann-Roch theorem for algebraic stacks proved by B. Toen [To].

Let $H$ be a finite subgroup of $G$. The natural map $R(G / H) \rightarrow R(G)$ is finite and injective. Let $\bar{I}$ be the augmentation ideal of $R(G / H)$. Consider the natural map $R(G / H) \rightarrow R(G)$.

\section{Lemma 5.2.}

$$
(*) \bar{I} \otimes_{R(G / H)} R(G)=\bigcap_{Q \in \mathcal{H}} Q
$$

where $\mathcal{H}=\{Q \in \operatorname{Spec} R(G) \mid H \supset \operatorname{Supp} Q\}$.

Proof of Lemma 5.9. Identify $R(H)=K^{G}(G / H)$. Since $G$ is abelian we have an isomorphism

$$
R(H)=K^{G}(G / H) \simeq K^{G / H}(G / H) \otimes_{R(G / H)} R(G)
$$

Since $\bar{I}$ is defined as the kernel of the augmentation map $R(G / H) \rightarrow \mathbb{Z}$ the right hand side of equation $\left(^{*}\right)$ is just $R(G) /\left(\bar{I} \otimes_{R(G / H)} R(G)\right)$. Hence $\bar{I} \otimes_{R(G / H)} R(G)=\operatorname{ker}(R(G) \rightarrow R(H))$. The lemma now follows from [Sega], Prop 3.7] and [Tho5, Prop 1.2]. 
Proof of Theorem 5.9. By the localization theorem for diagonalizable group schemes $G^{G}(X)_{P_{i}} \simeq G^{G}\left(X^{i}\right)_{P_{i}}$. Since $H_{i} \subset G$ acts trivially on $X^{i}$ we have [Tho1, Lemma 5.6]

$$
G^{G}\left(X^{i}\right) \simeq G^{G / H_{i}}\left(X^{i}\right) \otimes_{R\left(G / H_{i}\right)} R(G) .
$$

Let $\bar{I}_{i}$ be the augmentation ideal of $G / H_{i}$. Then the previous lemma implies that $P_{i}$ is a component of $\bar{I}_{i} \otimes_{R\left(G / H_{i}\right)} R(G)$. Thus,

$(*) \quad\left[G^{G / H_{i}}\left(X^{i}\right) \otimes_{R\left(G / H_{i}\right)} R(G)\right]_{P_{i}}=\left[G^{G / H_{i}}\left(X^{i}\right)_{\overline{I_{i}}} \otimes_{R\left(G / H_{i}\right)} R(G)\right]_{P_{i}}$.

Thus, by the Riemann-Roch isomorphism of Corollary 5.1 we have

$$
\left[G^{G / H_{i}}\left(X^{i}\right)_{\bar{I}_{i}} \otimes R\left(G / H_{i}\right) R_{G}\right]_{P_{i}} \simeq\left[C H_{G / H_{i}}^{*}\left(X_{i}\right) \otimes R\left(G / H_{i}\right) R_{G}\right]_{P_{i}}
$$

and the first statement follows.

As noted in Remark 5.1 there is an ideal $J \subset R(G)$ supported at a finite number of points such that $J G^{G}(X)=0$. Hence, $G^{G}(X) \simeq$ $G^{G}(X) \otimes_{R(G)} R(G) / J$. Then $J=Q_{1} \cap Q_{2} \ldots \cap Q_{k}$ with $Q_{i}$ a $P_{i}$-primary ideal. By the Chinese remainder theorem

$$
R(G) / J \simeq \prod_{i=1}^{r} R(G) / Q_{i}=\prod_{i=1}^{r}(R(G) / J)_{P_{i}}
$$

so

$$
G^{G}(X) \simeq \prod_{i} G^{G}(X)_{P_{i}}
$$

and the second statement follows.

\section{More on COMPletions}

There are other natural completions of $G^{G}(X)$ and $C H_{G}^{*}(X)$ besides those of Section 2. The purpose of this section is to prove that the different definitions give isomorphic completions. As an application of these results, we will prove a special case of a conjecture of Köck. To begin, we fix an embedding of $G$ into $G L_{n}$. Then $G^{G}(X)$ is an $R\left(G L_{n}\right)$-module.

Recall that $\widehat{G^{G}(X)}$ was defined to be completion of $G^{G}(X)$ along the augmentation ideal $I$ of $R\left(G L_{n}\right)$, and $\widehat{C H_{G}^{*}(X)}$ the completion of $C H_{G}^{*}(X)$ along the augmentation ideal of $A_{G}^{*}(p t)$. We will refer to these as the "point" completions because they are defined using ideals in the equivariant groups of a point.

Let $I_{X} \subset K^{G}(X)$ denote the augmentation ideal, i.e., the ideal of virtual vector bundles of rank 0 , and let $\widetilde{G^{G}(X)}$ denote the completion of $G^{G}(X)$ along $I_{X}$. Let $J_{X} \subset A_{G}^{*}(X)$ denote the augmentation ideal, and $\widetilde{C H_{G}^{*}(X)}$ the completion of $C H_{G}^{*}(X)$ along $J_{X}$. We will refer to 
these as the " $X$ " completions because they are defined using ideals in the equivariant groups of $X$. In the proof below it will be necessary to distinguish between ideals corresponding to different groups. We will use a subscript to indicate this, e.g., $I_{X, G} \subset K^{G}(X)$.

Note that the $X$-completions only depend on $G$ and $X$, while a priori, the point completions depend in addition on an embedding of $G$ into $G L_{n}$. The next theorem will imply that the point completion is independent of the embedding.

The main result of this section is that the point and $X$-completions are isomorphic.

Theorem 6.1. (a) The $I$-adic and $I_{X}$-adic topologies on $G^{G}(X)$ coincide. Hence we have an isomorphism of completions

$$
\widehat{G^{G}(X)} \simeq \widehat{G^{G}(X)}
$$

(b) The J-adic and $J_{X}$-adic topologies on $C H_{G}^{*}(X)$ coincide. Hence we have an isomorphism of completions

$$
\widehat{C H_{G}^{*}(X)} \simeq \widehat{C H_{G}^{*}(X)}
$$

Proof. (a) To show that the filtrations induced by powers of the ideals $I$ and $I_{X}$ induce the same topology, we must check two things. First, we must show that for any $n$, there exists an $r$ such that $I^{r} G^{G}(X) \subseteq$ $I_{X}^{n} G^{G}(X)$. This is clear because under the map $R\left(G L_{n}\right) \rightarrow K^{G}(X)$, the image of $I$ is contained in $I_{X}$, so we can take $n=r$. Second, we must show that for any $n$, there exists an $r$ such that $I_{X}^{r} G^{G}(X) \subseteq I^{n} G^{G}(X)$. As above we will do this in steps: first for $G=B$ the group of upper triangular matrices, then for $G=G L_{n}$, and finally for arbitrary $G$.

Suppose then that $B \subset G L_{n}$ is the group of upper triangular matrices. We use the notation of the proof of Theorem 2.2. We know there exists $m$ such that ker $k_{m} \subseteq I_{B}^{n} G^{B}(X)$. Since $I_{B}$ and $I$ generate the same topology on $R(B)$, we can assume ker $k_{m} \subseteq I^{n} G^{B}(X)$. So we must show that there exists $r$ such that $I_{X}^{r} G^{G}(X) \subseteq$ ker $k_{n}$, i.e., such that $I_{X}^{r} G^{B}\left(X \times U_{m}\right)=0$. Since $B$ acts freely on $X \times U_{m}$, we have $G^{B}\left(X \times U_{m}\right) \simeq G\left(X \times{ }^{B} U_{m}\right)$. Under this isomorphism,

$$
I_{X}^{r} G^{B}\left(X \times U_{m}\right) \subseteq \mathfrak{a}^{r} G\left(X \times{ }^{B} U_{m}\right)
$$

where $\mathfrak{a}^{r}$ denotes is the augmentation ideal of $K\left(X \times{ }^{B} U_{m}\right)$. By Lemma 2.5, $\mathfrak{a}^{r} G\left(X \times{ }^{B} U_{m}\right)=0$ for $r>>0$.

The analogous statement for Chow groups, that $J_{X}^{r} C H_{B}^{*}\left(X \times U_{m}\right)=$ 0 for $r>\operatorname{dim}\left(X \times{ }^{B} U_{m}\right)$, also holds. 
Assume now that $G=G L_{n}$. Then we have

$$
G^{B}(X) \simeq G^{G}(G / B \times X) \leftrightarrows G^{G}(X)
$$

where the two maps are $i_{\text {! }}$ and $i^{!}$. We have proved that there exists $r$ such that

$$
I_{X, B}^{r} G^{B}(X) \subset I^{n} G^{B}(X) .
$$

Hence

$$
i_{!}\left(I_{X, B}^{r} G^{B}(X)\right) \subset i_{!}\left(I^{n} G^{B}(X)\right)=I^{n} G^{G}(X)
$$

where the last equality follows by the projection formula. Now, we also have $i^{!}\left(I_{X, G}^{r} G^{G}(X)\right) \subset I_{X, B}^{r} G^{B}(X)$. Combining these facts, we see that

$$
i_{i} \imath^{!}\left(I_{X, G}^{r} G^{G}(X)\right) \subset I^{n} G^{G}(X) .
$$

Since $i_{i} i^{!}$is the identity $I_{X, G}^{r} G^{G}(X) \subset I^{n} G^{G}(X)$.

Finally consider the case where $G \hookrightarrow G L_{n}$ is any subgroup. By Proposition 3.2, there is an isomorphism

$$
G^{G}(X) \simeq G^{G L_{n}}\left(G L_{n} / G \times X\right) .
$$

[This is an isomorphism of $R\left(G L_{n}\right)$-modules, so under it, $I^{n} G^{G}(X)$ corresponds to $I^{n} G^{G L_{n}}\left(G L_{n} / G \times X\right)$. Moreover, $I_{X, G}^{r} G^{G}(X)$ corresponds to $I_{G L_{n} / G \times X, G L_{n}}^{r} G^{G L_{n}}\left(G L_{n} / G \times X\right)$. This isomorphism follows from an equivalence of categories, obtained from descent of $G L_{n} \times G$-equivariant sheaves on $G L_{n} \times X$ (cf. Proposition 3.3). Under descent, locally free sheaves correspond to locally free sheaves (of the same rank) EGA IV, Prop. 2.5.2].] Because of these correspondences, the theorem follows from the case where $G=G L_{n}$.

Corollary 6.1. If $H \subset G$, then the topology on $R(G)$ induced by the

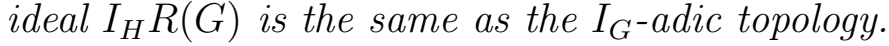

Proof. By embedding $H \subset G \subset G l_{n}$ it suffices to prove the result for $G \subset G l_{n}$. The corollary now follows by applying the theorem when $X=p t$.

Remark 6.1. In characteristic 0 this is the same as Segal, Corollary 3.9], but in characteristic $p$ the result is new. For a large class of group schemes over perfect fields Thomason [Tho2, Corollary 3.3] showed that $I_{G}$-adic and $I_{G L_{n}}$-adic topologies are the same on the mod $l^{\nu}$ equivariant $G$-theory localized at the Bott element.

Observe that the higher equivariant $K$-groups $G_{i}^{G}(X)\left(\operatorname{resp} . K_{i}^{G}(X)\right)$ are also modules over $R(G)$ and $K^{G}(X)$. Thus we can define completions of these groups with respect to the ideals $I_{X}$ and $I_{G}$. If $X$ is a regular scheme then $K_{i}^{G}(X)=G_{i}^{G}(X)$ and we can prove a corollary 
about higher $K$-theory as well. Part (b) proves a conjecture of Köck [Ko, Conjecture 5.6] for regular schemes.

Corollary 6.2. (a) If $X$ is a regular $G$-scheme then $\widehat{K_{i}^{G}(X)} \simeq \widehat{K_{i}^{G}(X)}$.

(b) Let $f: Y \rightarrow X$ be an equivariant proper morphism of regular $G$-schemes. Then the push-forward $f_{*}: K_{i}^{G}(Y) \rightarrow K_{i}^{G}(X)$ induces a map of completions $f_{*}: \widetilde{K_{i}^{G}(Y)} \rightarrow \widehat{K_{i}^{G}(X)}$.

Proof. The map $R(G) \rightarrow K_{i}^{G}(X)$ factors through $K^{G}(X)$. Since $K^{G}(X)=$ $G^{G}(X)$, by Theorem 6.1, the ideals $I_{X} \subset K^{G}(X)$ and the $I_{G} K^{G}(X)$ generate the same topology. Thus, $\widehat{K_{i}^{G}(X)} \simeq \widehat{K_{i}^{G}(X)}$ proving (a).

By the projection formula applied to the commutative triangle

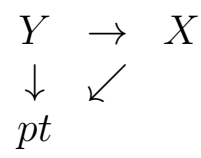

$f_{*}\left(I^{k} K_{i}^{G}(Y)\right)=I^{k} f_{*} K_{i}^{G}(Y) \subset I^{k} K_{i}^{G}(X)$. Hence, $f_{*}$ is continuous with respect to the $I$-adic topology, proving (b).

Remark 6.2. In its full form, Köck's conjecture asserts that if $X \rightarrow Y$ is any equivariant projective local complete intersection morphism then there is a push-forward

$$
f_{*}: \widetilde{K_{i}^{G}(Y)} \rightarrow \widetilde{K_{i}^{G}(Y)}
$$

of completions. This conjecture is quite subtle because (despite the suggestive notation) the completions are taken with respect to different ideals, and if $X$ and $Y$ are not regular, there is no obvious way of comparing the topologies.

\section{REFERENCES}

[AS] M. Atiyah, G. Segal, Equivariant K-theory and completion, J. Diff. Geom 3 (1969) pp. 1-18.

[BFM] P. Baum, W. Fulton, R. MacPherson, Riemann-Roch for singular varieties I.H.E.S. Publ. Math. 45 (1975), pp. 101-145.

[B] R. Bott, The index theorem for homogeneous differential operators in, Differential and Combinatorial Topology (A Symposium in Honor of Marston Morse) pp. 167-186, Princeton Univ. Press (1965).

[Br] M. Brion, Equivariant Chow groups for torus actions, Transform. Groups 2 (1997), pp. 225-267.

[CEPT] T. Chinburg, B. Erez, G. Pappas, M.J. Taylor, Riemann-Roch type theorems for arithmetic schemes with a finite group action, J. Reine Angew. Math. 489 (1997), pp. 151-187.

[EG1] D. Edidin, W. Graham, Characteristic classes in the Chow ring, J. Alg. Geo, 6 (1997) pp. 431-443. 
[EG2] D. Edidin, W. Graham, Equivariant intersection theory, Inventiones Math., 131 (1998) pp. 595-634.

[EGA II] A. Grothendieck, J. Dieudonné, Elements de Geometrie Algebraique, IV, Publ. Math. I.H.E.S. 8 (1961).

[EGA IV] A. Grothendieck, J. Dieudonné, Elements de Geometrie Algebraique, IV, Publ. Math. I.H.E.S. 24 (1965).

[FL] W. Fulton, S. Lang, Riemann-Roch algebras, Springer-Verlag (1985).

[Fu] W. Fulton, Intersection Theory, Ergebnisse, 3. Folge, Band 2, Springer Verlag (1984).

[Gi] H. Gillet, Intersection theory on algebraic stacks and Q-varieties, J. Pure Appl. Algebra, 34 (1984) 193-240.

[Kn] D. Knuston, Algebraic spaces, Lecture Notes in Math 203 , Springer Verlag (1971).

[Ko] B. Köck, The Grothendieck Riemann-Roch theorem in the higher $K$-theory of group scheme actions, Ann. Sci. École Norm. Sup. (4) 31 (1998), pp. 415-458.

[Segal] G. Segal, The representation ring of a compact Lie group, Publ. Math. I.H.E.S. 34 (1968) pp. 113-128.

[Serre] J-P Serre, Groupes de Grothendieck des schémas en groupes reductifs déployés, Publ. Math. I.H.E.S. 34 (1968) pp. 37-52.

[Tho1] R. Thomason, Lefschetz-Riemann-Roch theorem and coherent trace formula, Inv. Math. 85 (1986), pp. 515-543.

[Tho2] R. Thomason, Equivariant and topological K-theory, Duke Math. Journal, 53 (1986), pp. 795-825.

[Tho3] R. Thomason, Algebraic K-theory of group scheme actions, in Algebraic topology and algebraic K-theory (W. Browder editor), Annals of Math Studies 113 (1987), 539-563.

[Tho4] R. Thomason, Equivariant algebraic vs. topological K-theory, Duke Math. J., 56 (1988), pp. 589-636.

[Tho5] Une formule de Lefschetz en $K$-théorie Équivariante algébrique, Duke Math. J. 68 (1992), pp. 447-462.

[To] B. Toen, Riemann-Roch theorems for Deligne-Mumford stacks, preprint math.AG/9803076.

[T] B. Totaro, The Chow ring of the symmetric group, Contemp. Math., to appear.

[Vi] A. Vistoli, Equivariant Grothendieck groups and equivariant Chow groups in Classification of irregular varieties (Trento, 1990), Lecture Notes in Math., 1515, 112-133, Springer, Berlin, 1992.

Department of Mathematics, University of Missouri, Columbia MO 65211

Department of Mathematics, University of Georgia, Boyd Graduate Studies Research Center, Athens, GA 30602 